\title{
Electromagnetic Analysis of Fuzzy Controlled Active Magnetic Bearings
}

\author{
Theodoros Psonis ${ }^{1 *}$, Pantelis G. Nikolakopoulos ${ }^{2}$ andEpaminodas Mitronikas ${ }^{1}$.
}

${ }^{1}$ Laboratory of Electromechanical Energy Conversion, Department of Electrical and Computer Engineering, University of Patras,26500 Rio-Patras, Greece

${ }^{2}$ Machine Design Laboratory, Department of Mechanical Engineering and Aeronautics, University of Patras,26500 Rio-Patras, Greece

\begin{abstract}
The main objective of the current paper is to present the electromagnetic and thermal analysis of an AMB which is controlled by a non-linear controller, taking into account the weight of the rotor. The system consists of a rotor and two pairs of electromagnets, each one placed on the vertical and horizontal axis respectively. A Fuzzy PID controller [Fig. 2] is used to control a pair of electromagnets (vertical or horizontal axis) of the AMB system and the rotor. The magnetic field simulation has been implemented through the ANSYS software and the control settings have been simulated using MATLAB/SIMULINK software. The interaction between these two programs is presented throughout this study. In order to define the dynamic modelling of the overall system, it is considered that the rotating magnetic field of the motor does not affect the AMB. The coil of the magnetic bearings is fed by a controlled power source. As it has already been mentioned above, the non-linear control model is implemented through a Fuzzy PID Controller, while the system is disrupted by a white noise signal for the simulation of the disturbances taking place during normal operation.
\end{abstract}

\section{INTRODUCTION}

The increasing search in the field of electric motors has led to the need for development and possible replacement of the existing bearing technology. Bearings, even though a separate field of study, significantly affect the operation, the performance and the lifetime of the motor. In recent years, Active Magnetic Bearings (AMB) are considered to be one of the most significant fields of study aiming at the replacement of the existing bearing technology, such as rolling and journal bearings. The AMBs contribute to the improvement of the performance of the electric motors. These advantages are attributed to the elimination of the possible mechanical contacts between the rotor and bearings. The existence of friction influences the highest speed of rotor as well as the temperature increase in the bearing, which has a direct impact on its lifetime. The AMB aims to maintain, with the proper control system, the rotating axis centered, under every operating condition of the motor. The reduction of the mechanical losses is promoted through the absence of friction. Consequently, lubrication is not necessary. These features make the AMB's an ideal candidate for a wide spectrum of electromechanical applications. The absence of lubricants and lubrication seals gives the potential for redefinition of rotor dimensions and limits of strength. In the same way, these new bearings are ideally suitable for use in a variety of vacuum applications and sterile clean rooms [1-4]. These advantages have been provided a 
motivation for the development and study of this new technology. To this direction, the most important obstacle was the necessity for an appropriate control system of this new electromagnetic component. Many studies have been conducted with the use of linear controllers in linearized active magnetic bearing systems [5]. The need for better results has led to a further study of the system. Subsequent efforts have focused on either the use of complex models [6] or the use of nonlinear controllers. The use of nonlinear controllers in a linearized system has been provided optimum performance with better control. A comparison of a nonlinear fuzzy PID controller with a linear PID in a linearized system of a degree of freedom was presented throughout our previous work [7]. In this paper, the advantages of the use of a non-linear controller, even on a linearized system, are presented. The choice of the Fuzzy PID controller provides a variety of advantages, not only compared to a regular linear PID controller but also in comparison to other non-linear controllers. Specifically, the existence of a Fuzzy controller for the determination of the corresponding gains of a PID, gives the opportunity for reconfiguring the controller during system operation. Reconfiguration is based on the values of the rotor position error and its derivative. The existence of the fuzzy controller offers improved response of the system, in comparison to a linear controller. At the same time, the processing requirements and the additional computing time needed are shorter regarding other types of non-linear controllers. As it has been expected [8], the fuzzy controller provides shorter tuning time, accelerated response and reduced overshoot. The use of the fuzzy controller is associated with a more robust system. It can also be confirmed by comparing the controller with a classic PID theory that acts simultaneously on the same active magnetic bearing system. The two controllers are aiming to provide better control of the system in which external disturbances are present. In this paper, the electrical and magnetic behaviour of an electromagnetic system is presented, operating in two degrees of freedom. The control laws which were used to control the coils current on both degrees of freedom are based on the non-linear fuzzy PID controller theory.

\section{Model and equations}

The modelling and the simulations were performed using the ANSYS multiphysics software. Specifically, the system under examination consists of features which provide a greater accuracy in the dimensions and properties of the material simulated. Especially, the model contains the actual dimensions and properties of its materials. Additionally, the existence and the influence of the air is simulated, both in areas of special interest such as the air gap between the bearing and motor, and in areas of less interest such as outsides of bearing borders. Furthermore, better analysis and accuracy of calculations in the areas of intense interest, can be achieved by modifying the precision of our model calculations.

The proposed model consists of two pairs of electromagnets, one in vertical and the other one in horizontal position, and a cylindrical mass of iron which corresponds to the rotor of the machine (fig.1). This cylindrical mass has a diameter of $(D) 5 \mathrm{~cm}$ and a length $(l)$ of $5 \mathrm{~cm}$. It is assumed that the mass density of the shaft is $7.9 \mathrm{~g} / \mathrm{cm}^{3}$. The number of turns $(N)$ is 50 for each pole and the gap $(g)$ between the rotor and the inner wall of the bearing set is $0.5 \mathrm{~mm}$ [9-11].

The linearization of the above system has been achieved by considering that: the rotor is rigid and symmetrical, the gyroscopic effects as well as the magnetic flux are also neglected, the magnetism of iron is below the saturation level, the movement of the rotating mechanism in the axial direction is neglected and the leakage fluxes in the airgap are also neglected [5],[12]. In the rotor's equilibrium point as showed in fig 1, the only forces which act on the rotor are its weight $(W)$ and the force $\left(F_{l}\right)$ which is generated from the vertical pair of the electromagnets. The current required $\left(I_{\text {bias }}\right)$ for complete levitation of the shaft in 


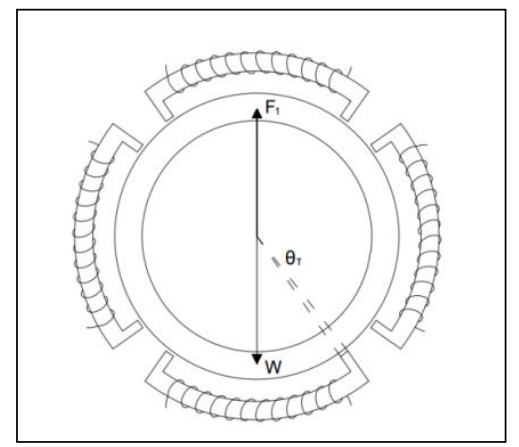

Fig. 1. A shaft and two pairs of electromagnets.

the initial equilibrium point is calculated by solving the following equations.

$$
F_{1}-W=0 \Rightarrow I_{\text {bias }}=\sqrt{\frac{(W \times 2 \times g)}{\left(\frac{N^{2} \times \mu_{0} \times S}{2 \times g}\right)}}
$$

Where $\mu_{0}$ is the Permeability of free space.

The cross-section area of the flux path $(S)$ is calculated as:

$$
S=l \times\left(\pi \times D \times \frac{\theta_{t}}{360}\right)=5.45 \times 10^{-4} \text { sq. meters }
$$

Where $\theta_{t}$ is the angle between shaft and electromagnet $\left(\theta_{t}=25 \mathrm{deg}\right)$.

$$
I_{\text {bias }}=4.91779 \mathrm{~A}
$$

The current value which is mentioned above, determines the suspension conditions of the system in an initial state of equilibrium.

In order to determine the current value in any other operational condition including external disturbances it is necessary to solve some additional equations such us the transfer function of close loop system, as it was presented in our previous work [7].

In the vertical axis, the characteristic equation of the open loop magnetic bearing system can be simply written as below:

$$
\frac{k_{i}}{m_{s} \times s^{2}-k_{x}}
$$

where $k_{i}$, is the force-current factor, $k_{x}$ is the force - displacement factor, $m_{s}$ is the mass of the shaft

The above characteristic equation represents a system which has no zeros and two poles $\mathrm{S}_{1,2}$ : 


$$
S_{1,2}= \pm \sqrt{\frac{k_{y y}}{m_{s}}}
$$

The positive pole of eq.(5) leads to an unstable system. The stability of the system is achieved through the presence of an appropriate control of the closed loop system.

\subsection{Configuration of fuzzy PID controller}

The controller that has been proposed to control the system for both equilibrium axes is a fuzzy PID controller. The rotor position error is defined as the first input of the controller (fig.2.) and the derivative of it as the second input. The outputs of the controller are the three gains of a PID controller. The use of the fuzzy controller helps the system to achieve more sensitivity even in small disturbances and helps also it discriminate between temporary and permanent errors. In fig. 3 the block diagram of the system with the fuzzy PID controller is presented.

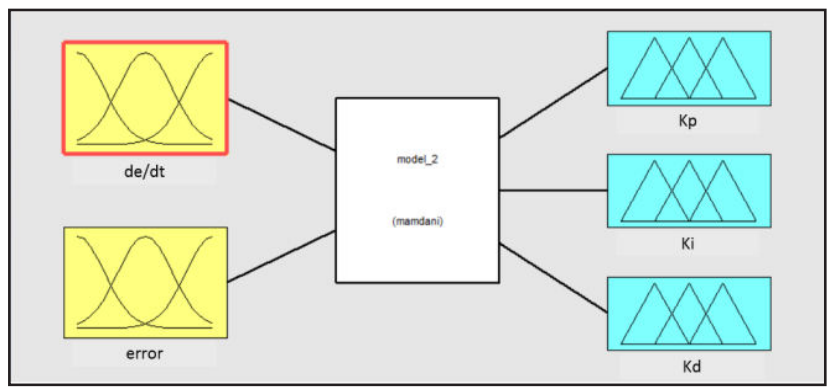

Fig.2. Structure of the fuzzy controller.

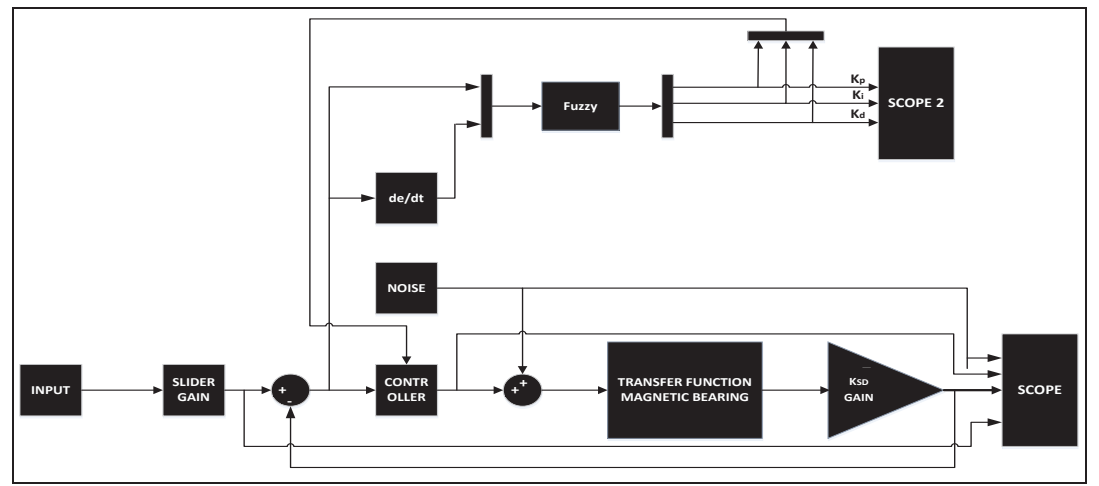

Fig.3. Block Diagram of the system with fuzzy PID controller.

The system with the fuzzy PID controller has a time-changing transfer function, as the gains of the controller change in each cycle of the system. The determination of the gains of the controller in each new system's operation cycle is determined by the values of the position error of the axis and its derivative is determined in the immediately preceding cycle of the system (outputs of the fuzzy controller). The variation of the gains (outputs of the fuzzy controller) is achieved through the fuzzy rules so that the system remains stable having all poles in the left (negative) half-plane. 


\section{Electromagnetic analysis}

This paper presents the electromagnetic analysis of our model with the existence of a suitable fuzzy PID controller. To this purpose, the ANSYS software was used to design and analyse the model (fig. 4). The controller and the disturbances that were acting on the system, were implemented using the MATLAB program. The parallel operation of these two programs enables both the monitoring of the changes in our controller's gains and the simultaneous electromagnetic analysis during external disturbances.

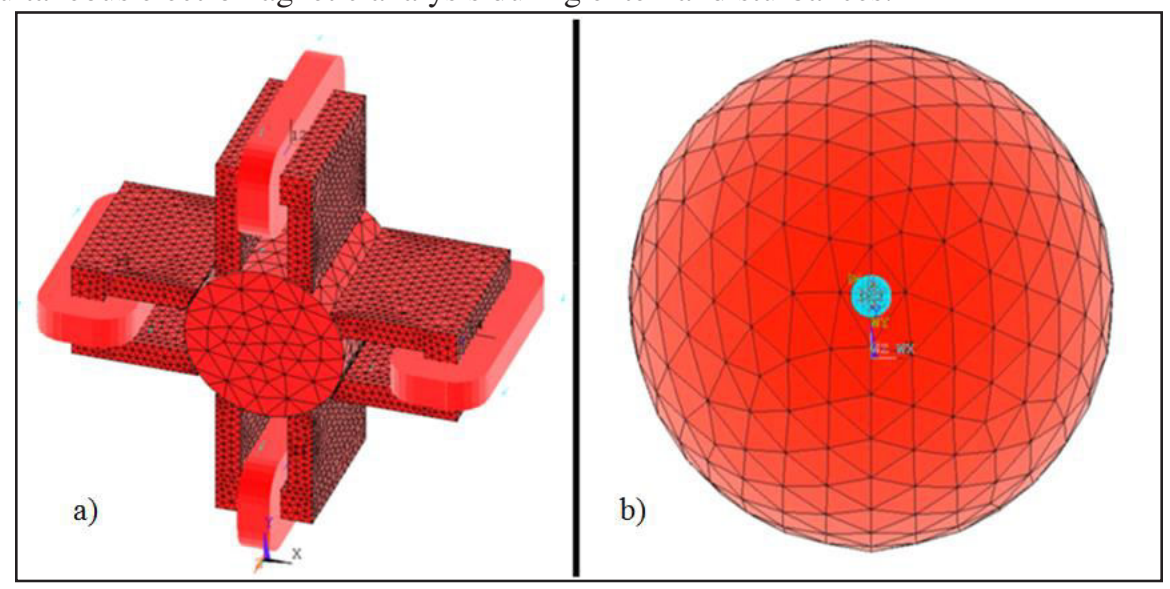

Fig.4.a) The shaft and two pairs of electromagnets, as designed in A, b) The mass of air which encloses the AMB.

The simulation model in ANSYS software is illustrated in fig.4a. The air mass that encloses the system is presented in fig.4b. In order to improve our calculations time and results precision, a different mesh structure and rectangular elements per surface has been used. The properties of materials are shown in Table 1. This specific modification is shown in the fig.5.

Table 1.Materials' properties.

\begin{tabular}{|c|c|c|c|c|}
\hline Material & $\begin{array}{c}\text { Number of } \\
\text { material in fig.5a }\end{array}$ & $\begin{array}{c}\text { Number of } \\
\text { nodes/vol. }\end{array}$ & $\begin{array}{c}\text { Relative } \\
\text { Permeability } \\
(\mathrm{H} / \mathrm{m})\end{array}$ & $\begin{array}{c}\text { Resistivity(oh } \\
\mathrm{m} \mathrm{m})\end{array}$ \\
\hline $\begin{array}{c}\text { Bearing's } \\
\text { Iron }\end{array}$ & 4 & 53327 & 1000 & $1 \times 10^{-6}$ \\
\hline Airgap & 1 & 213311 & 1 & $1 \times 10^{7}$ \\
\hline Rotor & 2 & 213311 & 1000 & $1 \times 10^{-6}$ \\
\hline
\end{tabular}




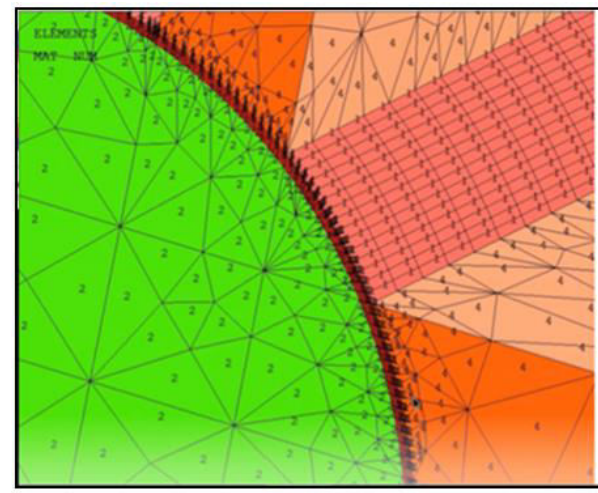

(a)

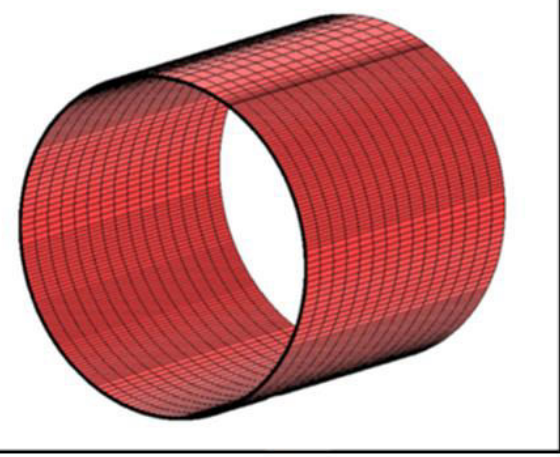

(b)

Fig.5.a) Different meshing structure per surface (rotor'2'- airgap'1'-bearings'4'), b) Airgap's meshing structure.

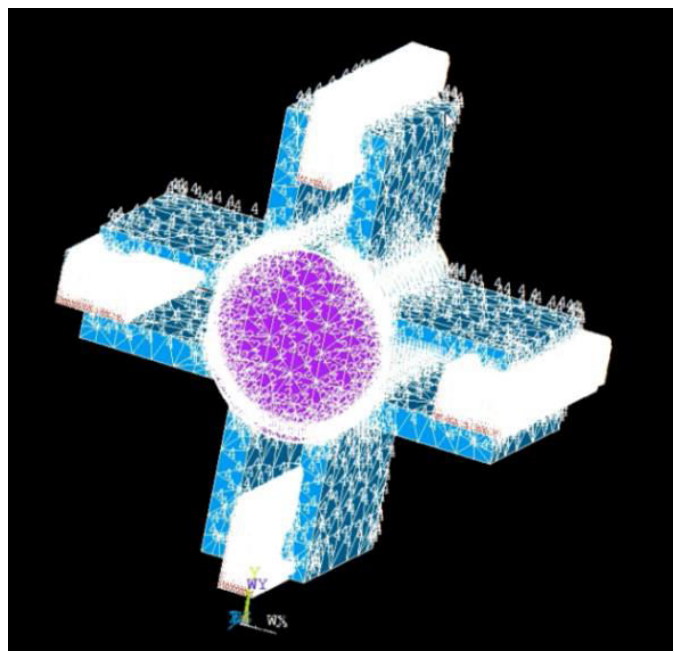

Fig.6.Av overview of meshing structureof the model.

The areas which need more in-depth study are the airgap between the bearing and the rotor and the areas between the bearings and the coils. In these areas, either high values of magnetic force (in the areas between the bearings and the coils) or high values of magnetic field and temperatures (in the airgap) could possibly appear. Additionally, the area of the airgap is changed depending on the position of the rotor. To this end, in cases of a rotor bearing system, which is an ongoing project for the authors, other dynamical phenomena such as gyroscopic effects, could also appeared in the for mentioned areas. All the above lead to the need to create a more detailed model of the bearing. For this reason, in order to increase the analysis accuracy and optimize the calculations, we formed a different meshing structure for these elements (fig.5b). The creation of 10 layers of the mesh for an airgap of $0.5 \mathrm{~mm}$ length provides a very good analysis with acceptable computational cost. To calculate the distribution of the electromagnetic field and the magnetic forces which exerted on the shaft, it is necessary to input data in the simulation model (fig.6). This data, which are the currents of each coil, come from the controller simulation implemented in MATLAB/SIMULINK. The controller needs, as an input, the previous position of the rotor, which is received from the AMB simulation out, come at the previous circuit's operation. 
The required time that ANSYS-MATLAB needed to calculate currents values based on the position of the rotor at the previous time for our system is $\tau=1 \times 10^{-3} \mathrm{sec}$.

Fig.7(a-b), shows the magnetic field of the system for time $t_{0}$. In this particular time the rotor levitates motionless at the equilibrium position in which the forces act from the four AMB's fields.

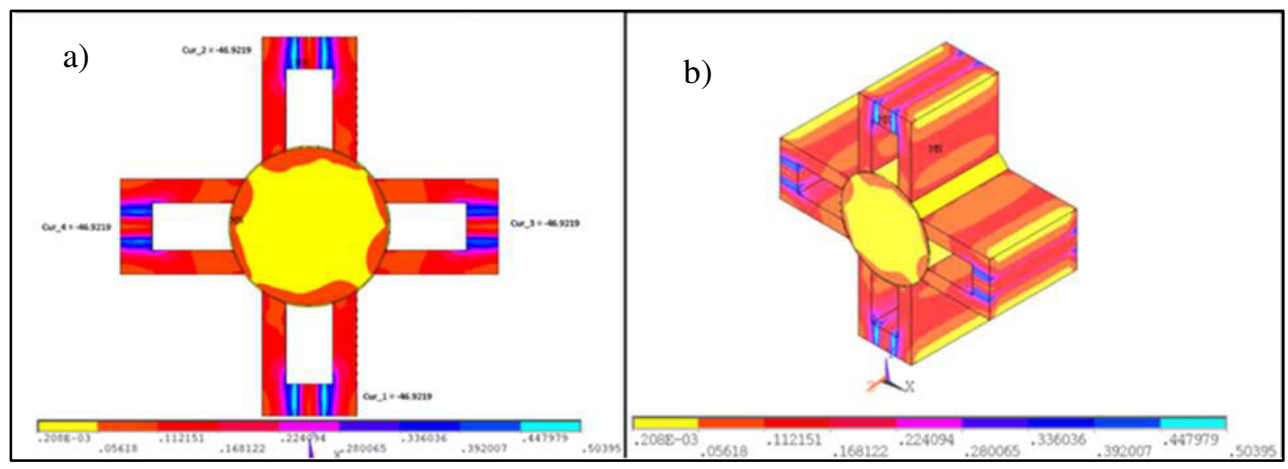

Fig.7.Magnetic flux densities $(\mathrm{T})$ for $\mathrm{t}_{0} \mathrm{a}$ ) frond viewof bearing, b) side viewof bearing.

Fig. 8 shows the magnetic poles of the bearings. At the time $t_{l}=t_{0}+\tau$, a disturbance is exerted on the shaft that results in its displacement from the equilibrium position by $y_{1}=3.4984 \times 10^{-04} \mathrm{~m}$. in the vertical axis. The controller calculates the required currents to restore the system into the equilibrium position. These currents values are used as inputs in the AMB's coils for the next step of magnetic field solution.

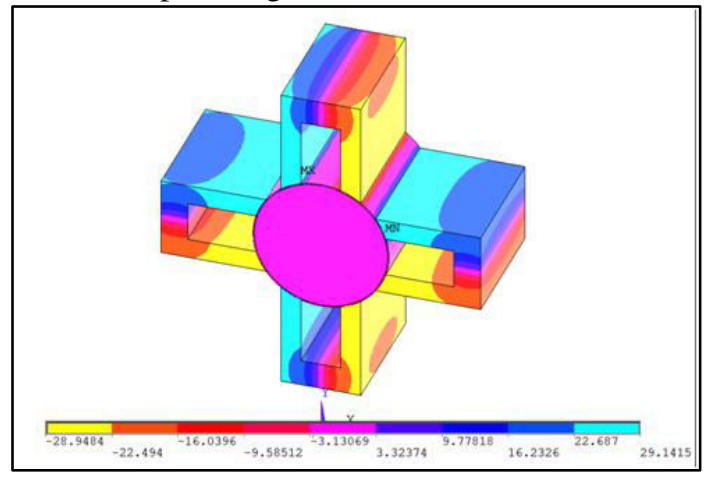

Fig.8.Magnetic scalar potential for $t_{0}$ (quantities indicated at the color bar are measured in A).

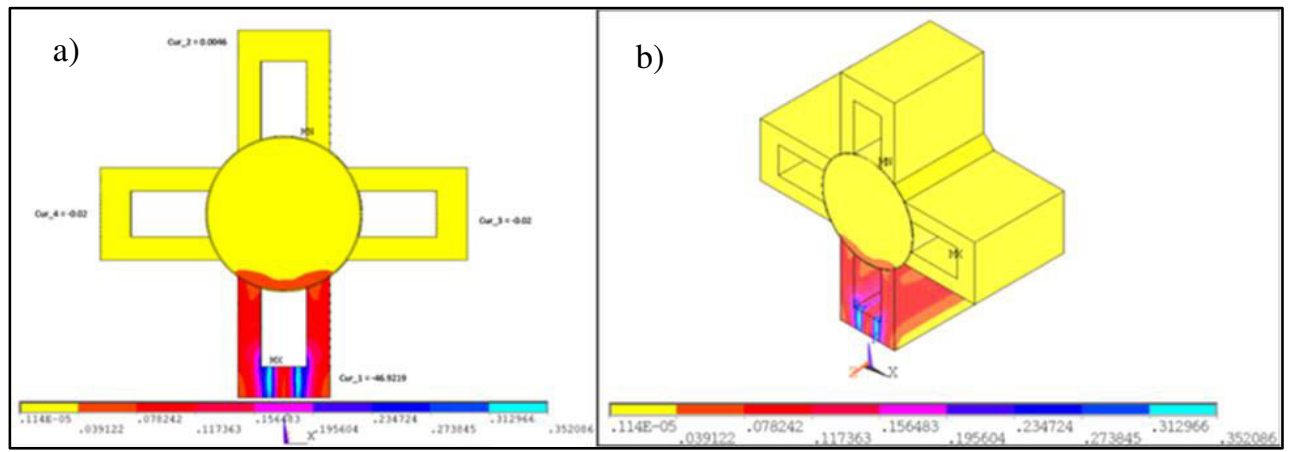

Fig.9.Magnetic flux densities (T) for $t_{2}=t_{1}+\tau$ : a) frond viewof bearing, b)side viewof bearing. 


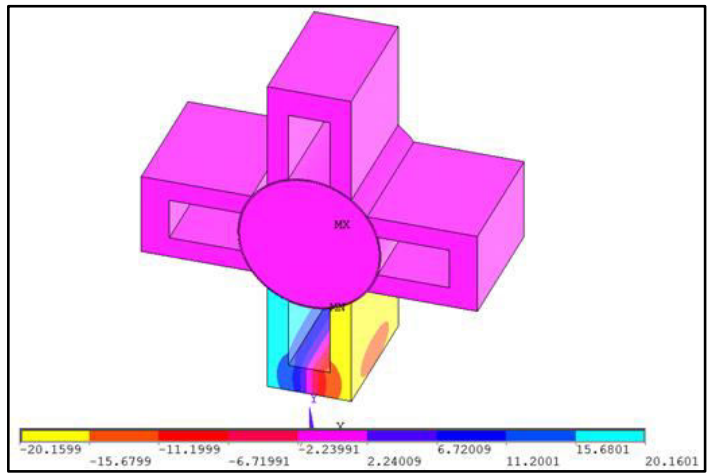

Fig.10. Magnetic scalar potential for $t_{2}=t_{1}+\tau$ (quantities indicated at the color bar are measured in $\left.\mathrm{A}\right)$.

Fig.9and 10 show the magnetic fields and magnetic poles of the system for the new values of the currents of the coils at time $t_{2}=t_{1}+\tau$. At time $t_{2}$, after imposing the above field $\left(t_{l}\right)$, the rotor is at the position $y_{2}=0,70671088 \times 10^{-08} \mathrm{~m}$. in the vertical axis.

The controller calculates the currents required for the new rotor's position. Fig. 11 and 12 present the magnetic fields and the magnetic poles of the system, regarding the new values of coil currents at time $t_{3}=t_{2}+\tau$.

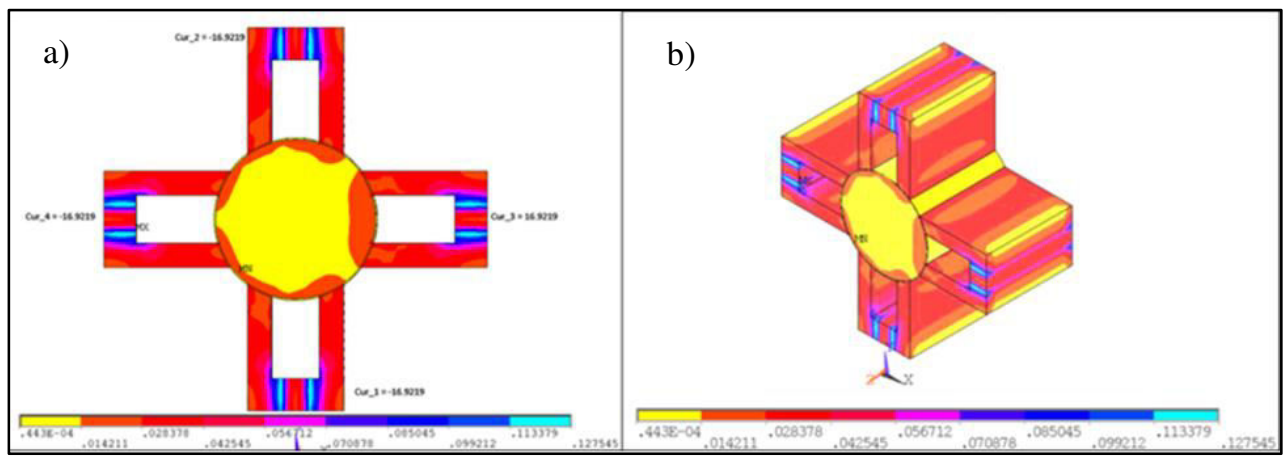

Fig.11.Magnetic flux densities (T) for $t_{3}=t_{2}+\tau$.a) Frond viewof bearing, b) Side viewof bearing.

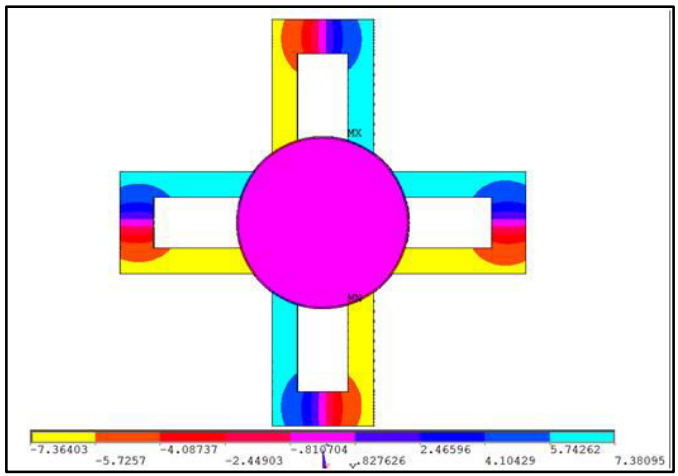

Fig.12. Magnetic scalar potential for $t_{3}=t_{2}+\tau \alpha$ (quantities indicated at the color bar are measured in A). 
Results such as those illustrated in fig. 7 to 12 , validate the correct operation of the fuzzy controller. In addition, fig.7, 9, and 11, derived by the magnetic field simulation, show the areas where the values of the magnetic field are close to the saturation area of the bearing core materials. These areas are quite narrow (the red areas) and close to the regions where the windings of the coils are placed. The size and the location of these particular areas are an indication to use linear properties (for example, we set a linear magnetic permeability for the bearing's iron, and we did not take the non-linear B-H loop of this material). Consequentially, the use of linear properties for the materials limit the computational requirements and the operation time of our model, without the need of more precision in the calculationsfor areas of immediate interest like the airgap. Elimination of the latter areas requires the use of non-linear properties, for example, non-linear B-H loop of material and additional iterative calculation methods. This would increase the computational requirements and the operation time of our model.

\section{Conclusion}

The magnetic analysis of a magnetic bearing system which is controlled by a Fuzzy PID controller has been presented throughout this paper. The above analysis provides better calculation in terms of computational time and results accuracy, as well as visualization of the system and its control possibility. The forces acting on the axis as well as the ones acting in other parts of the system, such as in the airgap, are introduced in the current paper. The obtained results confirm the ability of our controller to stabilize the rotor at the equilibrium point throughout the duration of the disturbances. Simultaneously, the power values and thus the energy required for the controller operation remains in a tolerable level. These results are considerably encouraging for further development of an AMB applied to electric motors.

\section{References}

1. G. Schweitzer and E.H. Maslen. Magn. B. Th., D. \& App. to Rot. Mach., Springer, (2009).

2. S.-L. Chen, S.-Y. Hsu, P.-L. Wang and D. Jaung, A f. St. of B. M. B. on Th.-P. AMB, International Journal of Automation and Smart Technology, 2, pp. 1-8, (2012).

3. L. Dong and S. You, Ad. C. of an AMB with ex. dist., ISA Transaction, 53, pp. 1410-1419, (2014).

4. A. Chiba, T. Fukao and O. Ichikawa, Mag. B. and B. Dr., Elsevier, (2005).

5. D. Polajžer, J. Ritonja, G. Ŝtumberger, D. Dolinar and J. Lecointe, Dec. PI/PD pos. c. for AMB, Electrical Engineering (ArchivfürElektrotechnik), 89, pp. 53 - 59, (2006).

6. M. G. Farmakopoulos, E. K. Loghis, P. G. Nikolakopoulos, N. I. Xiros, C. A. Papadopoulos, Mod. \& C. of the El. Act. S. of an Act. Hydr. J. B. (AHJB), ASME 2014 International Mechanical Engineering Congress \& Exposition, (2014).

7. T.K. Psonis, E.D. Mitronikas, P.G. Nikolakopoulos, Comp. of PID and Fuzzy PID C. for a Lin. Magn. B., Tribology in Industry, 39, pp. 340-348, (2017).

8. S.-C. Chen, V.-S. Nguyen, D.-K. Le, T.-H.-N. Nguyen, N. C. of an AMB S. Ach. Us. a Fuzzy C. with Rad. Fun. N. Net., journal of Applied Mathematics, (2014).

9. W. de Boer, AMB: Mod. \& cont. of a f. degr. of fr. R., M.Sc. Thesis, Eindhoven University of Technology , (1998).

10. G. Schweitzer. AMB - ch. \& L., 6th Internat.IFToMM Conf. on Rotor Dynamics, pp. 114,(2002).

11. B. Polajžer, D. Dolinar, G. Štumberger, J. Ritonja, B. Grčar and K. Hameyer, Des. of Hor. Sh. AMB S., 2nd International seminar on Vibrations and acoustic noise of electric machinery, pp. 51-55, (2012).

12. M.K. Habib and J. Inayat-Hussain, Fuzzy Log. B. C. of Rot. M. in AMB, Cybernetics and Intelligent System, IEEE, 2, pp. 1219-1225, (2004). 